\title{
Blends of L-tyrosine based polyurethanes for biomaterial applications
}

\author{
D. Sarkar, J-C. Yang, N. Klettlinger, S. T. Lopina* \\ Department of Chemical \& Biomolecular Engineering, The University of Akron, Akron, OH 44325-3906, USA
}

Received 14 June 2007; accepted in revised form 16 September 2007

\begin{abstract}
Three polyurethane blends were made from segmented polyurethanes synthesized from desaminotyrosyl tyrosine hexyl ester (DTH), an L-tyrosine based diphenolic dipeptide, as chain extender. The soft segment of these polyurethanes is either polyethylene glycol (PEG) or polycaprolactone diol (PCL) and the diisocyanate is hexamethylene diisocyanate (HDI). The blends were developed to investigate the effect of varying composition on the overall physical, thermal, mechanical, surface and degradation properties of the material. The characterization results show that the properties of these blended materials can be controlled by adjusting the composition. Blends with increased PEG based polyurethane exhibited more water absorption and higher degradation characteristics. With increasing PCL based polyurethane, the mechanical properties of the blends were improved although the blends were relatively more amorphous in nature. The L-tyrosine based polyurethane blends hold the potential for use in different biomaterial applications.
\end{abstract}

Keywords: polymer blends and alloys, L-tyrosine, polyurethane, poly(caprolactone), poly(ethylene glycol)

\section{Introduction}

Polyurethanes are an important class of biomaterials due to their excellent physical properties and relatively good biocompatibility [1]. Segmented polyurethanes synthesized from various polyols, diisocyanates and chain extenders can be structurally manipulated to achieve a wide range of properties suitable for various biomaterial applications [2]. The properties of segmented polyurethanes are determined by both the soft segment comprised of polyol and the hard segment comprised of diisocyanate and chain extender with the main contribution coming from the soft segment. The use of polyurethanes as tissue engineering material has received a great deal of attention due to its unique combination of physicomechanical properties and degradability [3]. Polyurethanes with polyester soft segment e.g. polycaprolactone (PCL) and polyether soft segment e.g. polyethylene

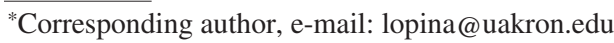

(C) BME-PT and GTE glycol (PEG) are mainly used for tissue engineering applications [3-5]. PCL based polyurethanes are relatively hydrophobic and have slow degradation rates whereas PEG based polyurethanes are highly hydrophilic and therefore show enhanced rates of degradation [3]. But in terms of mechanical properties, PEG based polyurethanes possess poorer moduli and ultimate stress compared to PCL based polyurethanes [5]. Thus PEG based polyurethanes lack the structural integrity required for tissue engineering scaffold formation. Blending and copolymerization are the most commonly used techniques to combine the properties of individual polymers $[6,7]$. However, blending is easier than the preparation of copolymers to obtain the advantageous properties of the constituent polymers [5]. Recently, we developed polyurethanes for biomaterial applications from an L-tyrosine based chain extender, desaminotyrosyl tyrosine hexyl ester (DTH) [8]. The polyols used for these poly- 


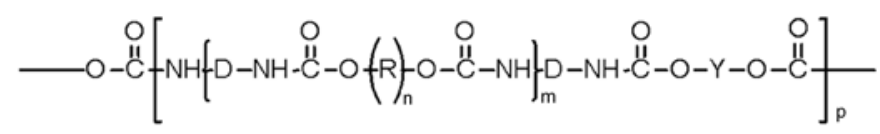

Polyurethane
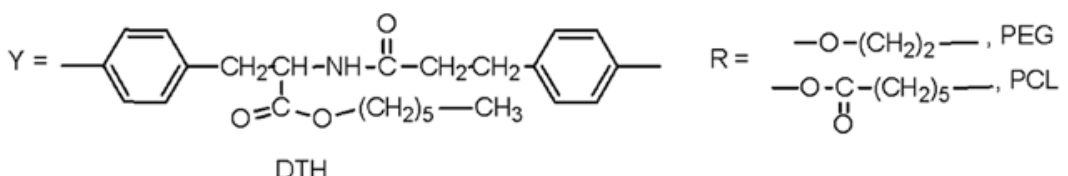

$\mathrm{D}=-\left(\mathrm{CH}_{2}\right)_{6}-$

$\mathrm{HDI}$

Figure 1. Structure of L-tyrosine based polyurethanes with PEG or PCL soft segment, HDI as diisocyanate and DTH as chain extender

urethanes are either PEG or PCL and the diisocyanate is hexamethylene diisocyanate (HDI). These polyurethanes have a wide range of physicomechanical properties that are suitable for tissue engineering applications. The structures of the polyurethanes are shown in Figure 1. The polyurethanes synthesized from PEG exhibit poor mechanical properties and a high rate of degradation whereas PCL based polyurethanes have better mechanical properties but very slow degradation rates.

In this work, three blends of L-tyrosine based polyurethanes were fabricated by using different compositions of the two polyurethanes. Structural and morphological characterization of the blends was carried out by spectroscopic, thermal and microscopic techniques. In addition, contact angle, water absorption and hydrolytic degradation studies were done to investigate the applicability of the systems for biomaterial applications.

\section{Experimental}

\subsection{Materials}

L-tyrosine, desaminotyrosine, Ethyl-N'-dimethylaminopropyl carbodiimide (EDC.HCl), polyethylene glycol(PEG), poly caprolactone diol(PCL), hexamethylene diisocyanate (HDI) were obtained from Sigma Aldrich, St. Louis(MO) USA. $n$-Hexanol, tetrahydrofuran, N-N'dimethylformamide (DMF), and chloroform were obtained from Fisher Scientific, Pittsburgh(PA) USA. The solvents were analytical grade and were used as received, unless otherwise mentioned. De-ionized water was used for all purposes.

Two different L-tyrosine based polyurethanes were synthesized from either polycaprolactone diol (PCL, $M_{w}=1250 \mathrm{~g} / \mathrm{mol}$ ) or polyethylene glycol (PEG, $M_{w}=1000 \mathrm{~g} / \mathrm{mol}$ ), as the polyol and hexa- methylene diisocyanate (HDI) as the diisocyanate. The chain extender was desaminotyrosyl tyrosine hexyl ester (DTH), a diphenolic dipeptide derived from L-tyrosine and its metabolite desaminotyrosine. PEG and PCL were dried in vacuum at $40^{\circ} \mathrm{C}$ for two days to remove water prior to the polymerization. The solvent N-N'dimethylformamide (DMF) was dried with calcium hydride and stored with molecular sieves for the polymerization reaction. All other chemicals were used as received. The detailed synthesis and characterization of the individual polyurethanes are described elsewhere [8].

Briefly, the chain extender DTH was synthesized by carbodiimide (EDC) mediated coupling of desaminotyrosine with $n$-hexyl ester of L-tyrosine in tetrahydrofuran as solvent at room temperature. The hexyl ester of L-tyrosine was prepared from the esterification of L-tyrosine with $n$-hexanol at $80^{\circ} \mathrm{C}$. The polyurethanes were synthesized by two step condensation polymerization reaction. PEG or PCL was reacted to HDI in the molar ratio of 1:2 at $110^{\circ} \mathrm{C}$ for three hours in DMF (as solvent) in the first step and was followed by the addition of DTH in the same molar proportion of PEG or PCL. The final step was carried out for 12 hours at $80^{\circ} \mathrm{C}$. Finally, the polymers were precipitated in cold concentrated aqueous solution of sodium chloride and were either centrifuged or filtered and dried in vacuum prior to any characterizations. The nomenclature for the polyurethanes used is: PEG-HDI-DTH and PCL-HDI-DTH, where PEG or PCL represents the soft segment and HDI and DTH represent the hard segment. The structures of the polyurethanes are shown in Figure 1. The polyurethanes were blended in three different weight ratios using chloroform as the solvent and films were cast by solvent evaporation. Typically 5\% (w/v) solutions of the polyurethanes were prepared in $10 \mathrm{ml}$ of chloro- 
Table 1. Formulation of blends

\begin{tabular}{|c|c|c|c|}
\hline Code & $\begin{array}{c}\text { Composition } \\
\text { (PEG-HDI-DTH/PCL-HDI-DTH) [wt \%] }\end{array}$ & $\begin{array}{c}\text { Weight } \\
\text { (PEG-HDI-DTH + PCL-HDI-DTH) [mg] }\end{array}$ & $\begin{array}{c}\text { Volume of solvent } \\
\text { (chloroform) [ml] }\end{array}$ \\
\hline PU1 & $100 / 0$ & 500 & 10 \\
\hline PU2 & $67 / 33$ & 500 & 10 \\
\hline PU3 & $50 / 50$ & 500 & 10 \\
\hline PU4 & $33 / 67$ & 500 & 10 \\
\hline PU5 & $0 / 100$ & 500 & 10 \\
\hline
\end{tabular}

form. Accurately weighed polymers $(500 \mathrm{mg}$ ) were dissolved in $10 \mathrm{ml}$ of solvent and allowed to form a homogeneous solution through constant stirring at room temperature for 24 hours. The polymer solutions were filtered through Teflon syringe filters to remove undissolved residue and were cast onto poly(tetrafluoroethylene) (PTFE) Perti dishes. The solvents were initially allowed to evaporate at room temperature followed by vacuum drying at $50^{\circ} \mathrm{C}$ for another 48 hours to remove the residual solvents. Films of about thickness $0.15 \mathrm{~mm}$ were obtained by this method. The nomenclature and summary of the blends are presented in Table 1.

\subsection{Measurements}

The blends were characterized by ${ }^{1} \mathrm{H}-\mathrm{NMR}$ and FTIR. NMR was carried out in a $300 \mathrm{MHz}$ Varian Gemini instrument with $d$-chloroform $(\delta=$ $7.27 \mathrm{ppm}$ as internal reference) and FT-IR analysis was performed with a Nicolet NEXUS 870 FT spectrometer for neat samples. Differential scanning calorimetry (DSC) was performed with a DSC Q100V7.0 Build 244 (Universal V3. 7A TA) instrument at a scanning rate of $10^{\circ} \mathrm{C} / \mathrm{min}$ from -80 to $250^{\circ} \mathrm{C}$. The tensile properties of the films were measured by Instron Tensile Testing Machine with a load cell of $100 \mathrm{~N}$ and cross-head speed of $100 \mathrm{~mm} / \mathrm{min}$ at room temperature. The sample dimension was $20 \mathrm{~mm} \times 6 \mathrm{~mm} \times 0.3 \mathrm{~mm}$ (approximately) with a free length of $10 \mathrm{~mm}$. For contact angle measurement, thin films of polymers were prepared on thoroughly cleaned and dried glass slides by dip coating the slides into the $5 \mathrm{wt} \%$ solution of polyurethane for 12 hours. The films were initially dried at room temperature for 24 hours followed by vacuum drying at $50^{\circ} \mathrm{C}$ for another 48 hours to remove the residual solvents. Static water contact angle was measured by sessile drop method using a Ramé-Hart goniometer at room temperature in an air atmosphere both in advancing and receding modes [4]. To measure water absorp- tion, circular samples were cut from dried films (diameter: $1.5 \mathrm{~cm}$ and thickness: $0.15 \mathrm{~mm}$ ) and immersed in $20 \mathrm{ml}$ of deionized water. At predetermined time intervals the hydrated samples were taken out and weighed after the surface water was blotted with Kimwipes. The water absorption was calculated on the basis of the weight difference of the film before and after swelling. The percentage of water absorption was calculated using Equation (1):

water absorption $[\%]=\frac{w_{2}-w_{1}}{w_{1}} \cdot 100$

where, $w_{2}$ and $w_{1}$ are the weight of sample films after and before being immersed in water, respectively. For hydrolytic degradation, similar circular samples (diameter: $1.0 \mathrm{~cm}$ and thickness: $0.15 \mathrm{~mm}$ ) were cut from dried films. The samples were incubated at $37 \pm 1^{\circ} \mathrm{C}$ in $10 \mathrm{ml}$ of phosphate-buffered saline (PBS; $0.1 \mathrm{M}, \mathrm{pH} 7.4$ ) containing $200 \mathrm{mg} \cdot \mathrm{l}^{-1}$ of sodium azide to inhibit bacterial growth in a sealed vial placed within a constant temperature water bath. Samples were taken at intervals, weighed for mass loss after drying under vacuum at $40^{\circ} \mathrm{C}$ for 2 days. The hydrolytic degradation was calculated from the weight loss [\%] using Equation (2):

weight loss $[\%]=\frac{w_{1}-w_{2}}{w_{1}} \cdot 100$

where, $w_{2}$ and $w_{1}$ are the weight of sample films after and before degradation, respectively. The SEM images were recorded on silver sputtered samples in Hitachi S2150 (Operating Voltage: $20 \mathrm{kV})$.

\section{Results and discussions}

\section{1. ${ }^{1} \mathrm{H}-\mathrm{NMR}$ and FT-IR characterizations}

The ${ }^{1} \mathrm{H}-\mathrm{NMR}$ spectra for all the blends are very similar due to the similarity in most of the proton 
environments. A representative spectrum for the blend (for PU3) is shown in Figure 2. Quantitative estimation by integrating a peak area is difficult due to similar chemical shift of the different protons present in soft and hard segment of the polyurethane [9]. Moreover the results were not

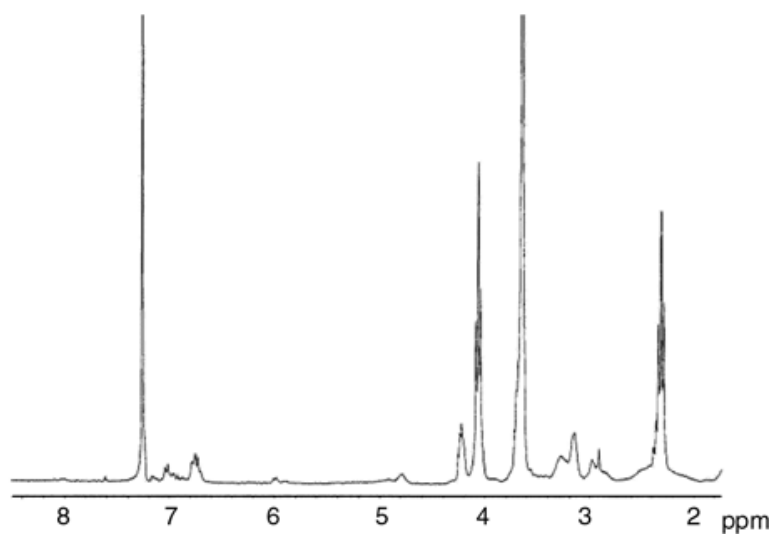

Figure 2. Representative ${ }^{1} \mathrm{H}-\mathrm{NMR}$ of polyurethane blend reproducible due to sample size and variation. Since the only difference is in the soft segment, two peaks were chosen that were characteristic of the PEG and PCL. The chemical shifts $(\delta)$ at $3.65 \mathrm{ppm}$ corresponding to methylene protons $\left(-\mathrm{CH}_{2}-\mathrm{CH}_{2}-\right)$ of the PEG soft segment and at $4.06 \mathrm{ppm}$ corresponding to methylene protons $\left(-\mathrm{CH}_{2}-\mathrm{O}-\mathrm{CO}-\right)$ of the PCL soft segment are integrated to estimate the relative contribution of the constituent polyurethane $[5,8]$. The interferences due to the peaks present at 4.25 and $3.70 \mathrm{ppm}$ from PCL based polyurethanes were taken into account for the calculation. The results of the integration are shown in Table 2 and indicate that the composition of the blends follows the general trends i. e. PU1 fraction decreases and PU5 fraction increases from PU2 to PU4. However, a significant deviation from the theoretical composition was observed for all the blends. This can be attributed to inhomogeneous
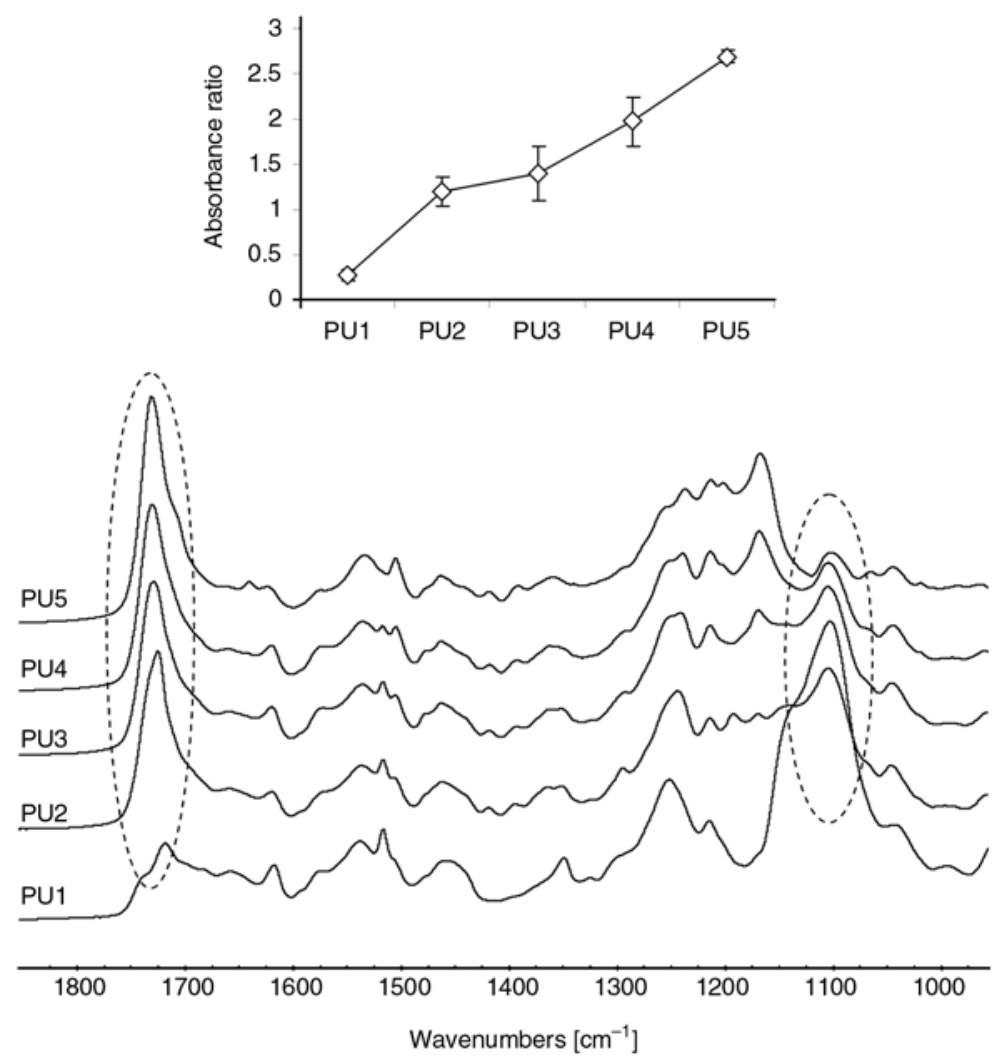

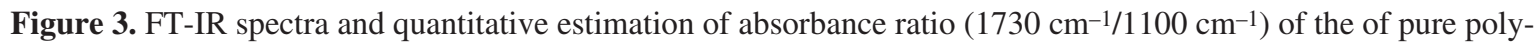
urethanes and blends (error bars represent standard deviation of measurement from 3 samples)

Table 2. Composition of polymer blends from ${ }^{1} \mathrm{H}-\mathrm{NMR}$

\begin{tabular}{|c|c|c|}
\hline Sample & $\begin{array}{c}\text { Theoretical ratio } \\
\text { (PEG-HDI-DTH/PCL-HDI-DTH) }\end{array}$ & $\begin{array}{c}\text { Observed ratio } \\
\text { (PEG-HDI-DTH/PCL-HDI-DTH) }\end{array}$ \\
\hline PU3 & $2: 1$ & $2.31: 1$ \\
\hline PU4 & $1: 1$ & $1.61: 1$ \\
\hline PU5 & $1: 2$ & $1.28: 2$ \\
\hline
\end{tabular}


mixing due to incompatibility, variation in sample size and similarity in the proton environment [9]. All the blends show considerably higher fraction of PEG based polyurethanes than the theoretical fraction which indicates some extent of immiscibility between the component polyurethanes.

The FT-IR spectra of the blends are shown with pure polyurethanes in Figure 3. The characteristic peak for PU1 is the absorbance at $1100 \mathrm{~cm}^{-1}$ corresponding to the aliphatic ether linkages $(\mathrm{C}-\mathrm{O}-\mathrm{C})$ present in the PEG soft segment and that for PU5 is the absorbance at $1730 \mathrm{~cm}^{-1}$ corresponding to the ester carbonyl linkage $(\mathrm{C}=\mathrm{O})$ present in the $\mathrm{PCL}$ soft segment. In addition, the spectra display characteristic urethane absorbance at $1713 \mathrm{~cm}^{-1}$, aromatic $\mathrm{C}=\mathrm{C}$ stretch at $1620 \mathrm{~cm}^{-1}$ (in DTH) and $\mathrm{C}=\mathrm{O}$ for amide I bonds at $1640 \mathrm{~cm}^{-1}$. The intensity of the absorbance due to ether linkage around $1100 \mathrm{~cm}^{-1}$ gradually decreases and that for $1730 \mathrm{~cm}^{-1}$ gradually increases from PU1 to PU5. This qualitatively suggests that the content of PEG based polyurethane is gradually decreasing from PU2 to PU4 while the content for PCL based polyurethane is increasing. A quantitative characterization of the blends was attempted by calculating the ratio of the absorbance for 1713 to $1100 \mathrm{~cm}^{-1}$ and the variation of the absorbance ratio is plotted against the composition in the inset of Figure 3. This estimation shows that ratio is least in PU1 and increases for the blends from PU2 to PU4 and is highest in PU5. This estimation qualitatively agrees that PU2 has highest fraction of PEG-HDI-DTH and PU4 has highest fraction of PCL-HDI-DTH.

\subsection{Differential scanning calorimetry}

The thermal characteristics of the blends were assessed by the differential scanning calorimetry (DSC). The DSC thermograms of the pure polyurethanes and the blends are presented in Figure 4. The glass transition temperature $\left(T_{g}\right)$ of PU1 is $-40^{\circ} \mathrm{C}$ and of PU5 is $-35^{\circ} \mathrm{C}$. Both PU1 and PU5 exhibit additional endotherms which are assigned as dissociation of ordered hard segment and melting of crystalline hard segment. No hard segment $T_{g}$ was observed for the pure polymers but PU5 exhibited soft segment melting endotherm at $-31^{\circ} \mathrm{C}$ [8].

$T_{g}$ for the blends is in between the range of -40 to $-35^{\circ} \mathrm{C}$. A shift in the glass transition temperature is

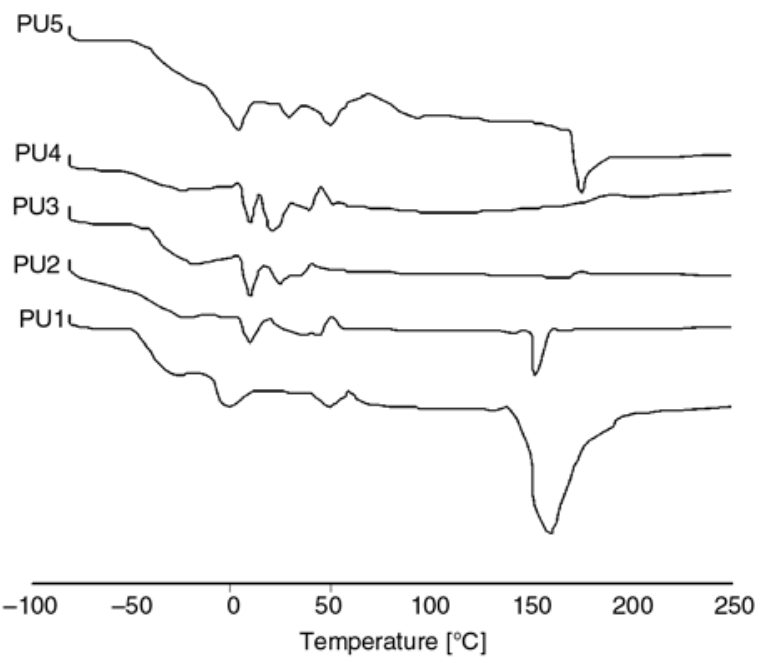

Figure 4. DSC thermograms of pure polyurethanes and blends

indicative of polymer miscibility but any appreciable shift in the $T_{g}$ for the blends is hard to detect within this small range [10]. However for PU2, which has higher fraction of PU1, the $T_{g}$ is close to $-40^{\circ} \mathrm{C}$ and for PU4, which has higher fraction of PU5, the $T_{g}$ is close to $-35^{\circ} \mathrm{C}$. The $T_{g}$ for PU3 which has equal fraction of PU1 and PU5 is also close to $-40^{\circ} \mathrm{C}$, indicating the dominance of PU1 fraction over PU5. The decrease in $T_{g}$ values with increasing PEG containing polyurethanes in consistent with results observed for blends of phenyl alanine based polyurethanes [9]. All the blends from PU2 to PU4 exhibit an endotherm around $10^{\circ} \mathrm{C}$ which is due to dissociation of short range order of the hard segment. But the endotherms observed due to dissociation of long range order in PU1 and PU5 around $50^{\circ} \mathrm{C}$ is only observed in PU2 and is present in PU3 as a broad endothermic transition and is absent in PU4. This indicates that increasing the amount of PU5 inhibits the formation of long range order of the hard segment. This feature is further substantiated by the absence of the hard segment melting endotherm in PU3 and PU4 which indicates that with higher PU5 content the hard segment is more amorphous in nature. Only PU2 with minimum PU5 fraction exhibits a melting endotherm at $152^{\circ} \mathrm{C}$. The absence of soft segment melting in PU1 and PU2 indicates that samples with more PEG content are amorphous in nature. The soft segment melting endotherm starts to appear in PU3. This feature shows that relatively crystalline PCL components show a melting endotherm. The soft segment melting endotherm for PU5 appears at $31{ }^{\circ} \mathrm{C}$ and for PU4 and PU3 at slightly lower tem- 
peratures around $25^{\circ} \mathrm{C}$. This feature shows that at some concentration, the presence of PEG helps to crystallize the PCL component. This feature is reported for similar systems [11]. In general, the $T_{g}$ values of the sample show that the polyurethanes are not completely miscible and a possible phase separation occurs due to incompatibility of hydrophilic PEG and hydrophobic PCL soft segment. However due to the chemical similarity of the hard segments, the hard segments of the constituent polyurethanes are likely to form an amorphous one phase domain as evident from the disappearance of hard segment melting endotherm of PU3 and PU4 [12].

\subsection{Mechanical properties}

The mechanical properties of the blends and the pure polyurethanes are summarized in Table 3 . The results show that the blend properties resemble the property of PU1. Even for the blends with comparatively higher PU5 content, i. e. PU3 and PU4, the properties are closer to pure polyurethane PU1. The reason for inferior mechanical properties of the blend PU3 and PU4 is mainly attributed to the distribution of the hard segment fraction of the polyurethane in the blend [13]. In presence of PCL soft segment, it is likely that hard segments do not have any long range order and are more amorphous in nature. The absence of the crystalline melting endotherm of PU3 and PU4 in the DSC analysis indicates that hard segments are not ordered and are random in distribution. The random distribution of the hard segment leads to inferior mechanical properties of the blends PU3 and PU4 in spite of higher content of PU5. Another possible exception is that phase separation between the polyurethanes is likely contributing to lower mechanical properties [13]. The phase separation can be due to several factors, with incompatibility between the hydrophilic PEG and hydrophobic PCL soft segment as the major one. The phase separation between the two phases is likely to constitute a continuous matrix of PU1 in which PU5 is dispersed as discrete domains. This gives a probable explanation for the result that in spite of having higher PU5 fraction, PU3 and PU4 exhibit relatively poorer mechanical properties.

\subsection{Contact angle}

The contact angle values both in advancing and receding modes are shown in Table 4 . The values are average of measurements from 6 readings taken from 3 replicates for each sample. The high contact angle values of the blends indicate that the surfaces are relatively hydrophobic. The hysteresis values of the blends are closer to pure PCL based polyurethane, i. e. PU5, which further supports that the blend surfaces are hydrophobic. The values show that the contact angle of the blend PU2 and PU3 is comparatively closer to PU5 in spite of having higher and equal fraction of PU1 respectively. This indicates a possible phase separation between the components of the polyurethanes with the PCL based polyurethanes migrating from the bulk to the surface [14].

\subsection{Water absorption}

The water absorption characteristic of the blends is plotted against time and shown in Figure 5 with along with the diagram showing the variation in water absorption (after 17 hours) with the change in

Table 3. Mechanical properties of the samples (mean $\pm \mathrm{SD}, n=5$ )

\begin{tabular}{|c|c|c|c|}
\hline Sample & Ultimate tensile strength [MPa] & Modulus of elasticity [MPa] & Elongation at break [\%] \\
\hline PU1 & $2.81 \pm 0.11$ & $3.75 \pm 0.21$ & $214 \pm 9$ \\
\hline PU2 & $3.35 \pm 0.25$ & $5.11 \pm 0.73$ & $285 \pm 57$ \\
\hline PU3 & $3.90 \pm 0.41$ & $6.11 \pm 0.95$ & $343 \pm 21$ \\
\hline PU4 & $4.21 \pm 0.29$ & $6.72 \pm 0.91$ & $361 \pm 64$ \\
\hline PU5 & $7.05 \pm 0.60$ & $17.98 \pm 0.68$ & $643 \pm 87$ \\
\hline
\end{tabular}

Table 4. Contact angle of the samples $(n=6)$

\begin{tabular}{|c|c|c|c|}
\hline Sample & Advancing contact angle (a) & Receding contact angle (r) & Hysteresis (a-r) \\
\hline PU1 & $33.0^{\circ}$ & $21.4^{\circ}$ & $11.4^{\circ}$ \\
\hline PU2 & $58.7^{\circ}$ & $33.7^{\circ}$ & $25.0^{\circ}$ \\
\hline PU3 & $68.7^{\circ}$ & $39.6^{\circ}$ & $29.1^{\circ}$ \\
\hline PU4 & $72.0^{\circ}$ & $42.3^{\circ}$ & $29.7^{\circ}$ \\
\hline PU5 & $75.0^{\circ}$ & $50.5^{\circ}$ & $24.5^{\circ}$ \\
\hline
\end{tabular}


composition of the blends. The water absorption values after 17 hours are not conclusive because of
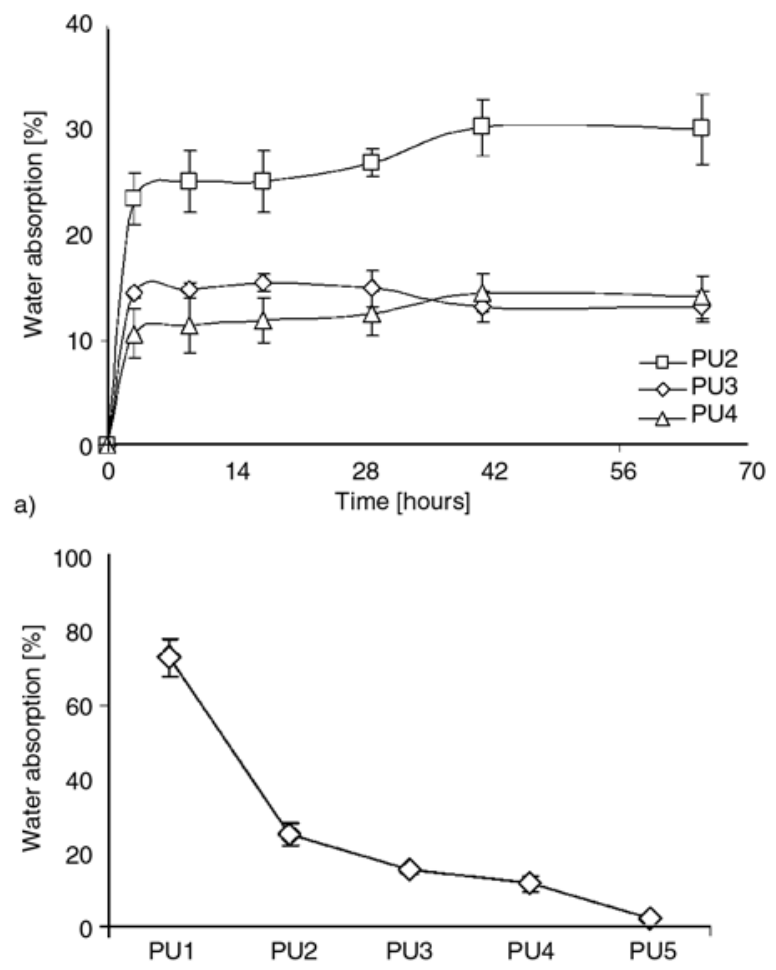

b)

Figure 5. a) Plot of water absorption of blends against time b) Comparison of water absorption of pure polyurethanes and blends after 17 hours (error bars represent standard deviation of measurement from 3 samples) weight loss due to degradation of the polymers. The water absorption of the blends increases from PU2 to PU4 with increasing content of PEG based polyurethane, i. e. PU1. As PEG is hydrophilic in nature, blends having higher PU1 content absorb more water. Blends PU4 and PU3 have water absorption characteristics similar to PU5 whereas PU2, having the highest fraction of PU1, has $25 \%$ water absorption compared to $73 \%$ for PU1. This relatively low water absorption is probably due to the surface characteristics of the PU2 which is predominantly hydrophobic with PCL based polyurethane phase separated and migrating to the surface. This migration is also evident from the contact angle measurements. The results presented follow the general trend that water uptake increases with increasing PU1, i. e. PEG based polyurethane content $[5,9]$.

\subsection{Hydrolytic degradation}

The hydrolytic degradation of the samples was measured by the weight loss and is shown in Figure 6 . The weight loss is higher with increasing PU1 content, indicating that increased water absorption leads to more hydrolytic degradation. The degradation of the blends decreases from PU2 to PU4 with decreasing PU1 content. Blend PU4,

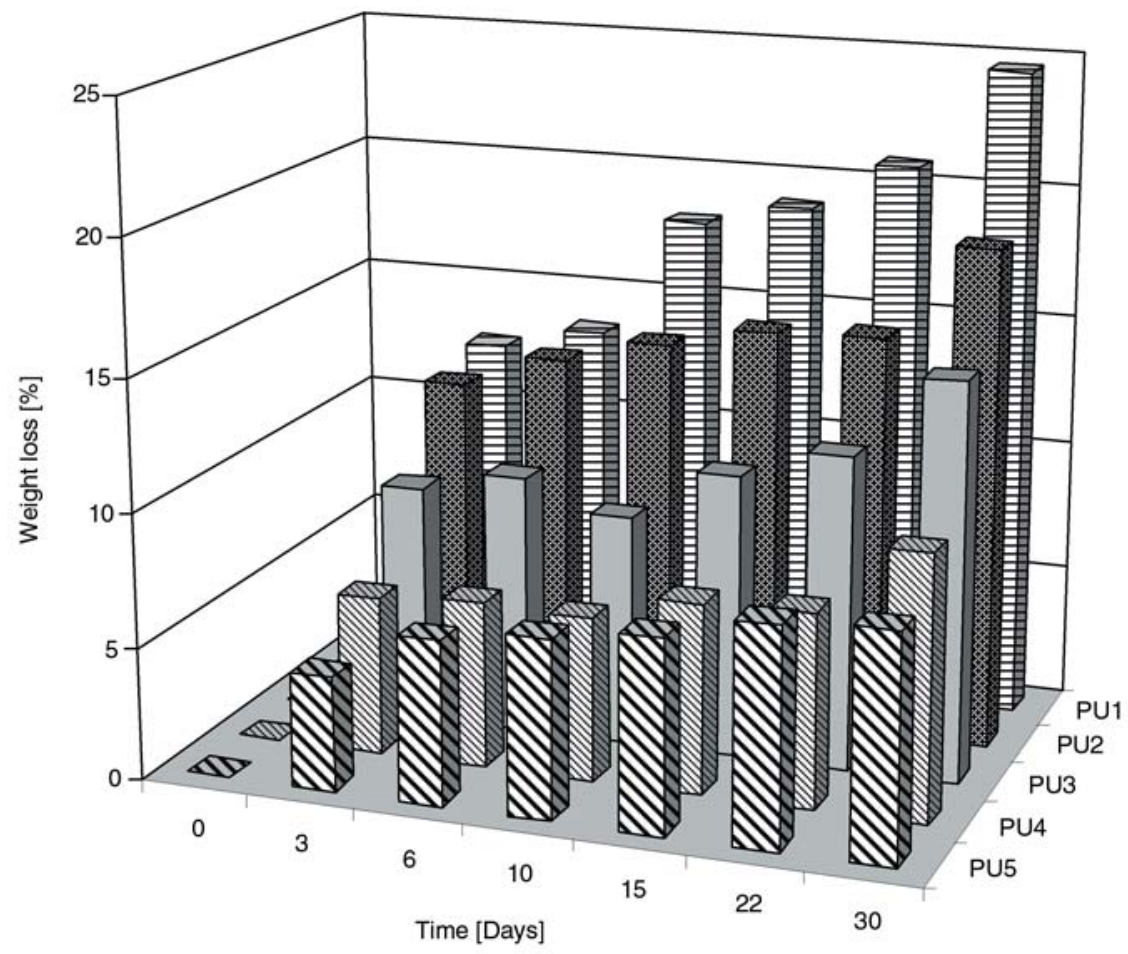

Figure 6. Weight loss of due to hydrolytic degradation for 30 day (results are average of 3 measurements) 
with 33 weight percent of PU1, has almost similar degradation characteristics compared to PU5. PU3 and PU2 have significantly higher degradation compared to PU4 in 30 days.

This indicates that the controlling factor in the degradation of the blends is the soft segment characteristics. The hydrophilic PEG component

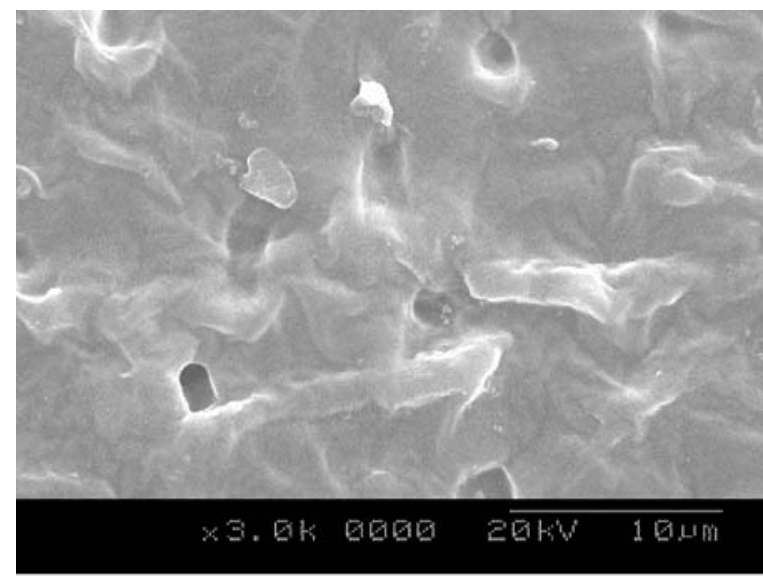

PU1

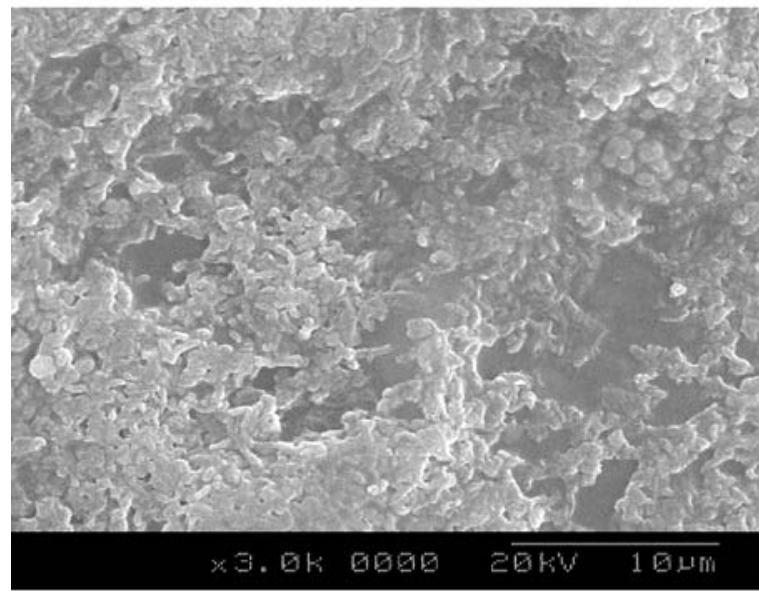

PU2 absorbs more water leading to degradation of the hydrolytically labile urethane, amide and ester linkages present in the polymer leading to more degradation. For all the samples the initial degradation was rapid followed by a significantly slower rate over the 30 day period. This is probably due to rapid and initial degradation of PEG based poly-

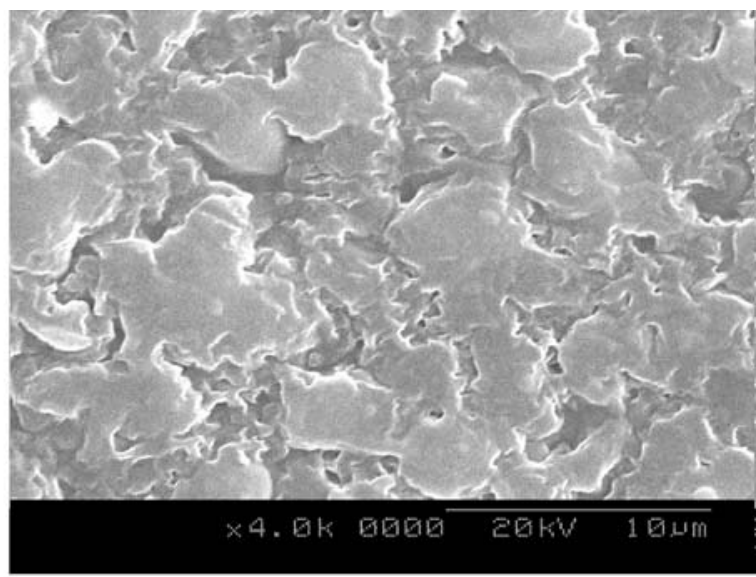

PU5

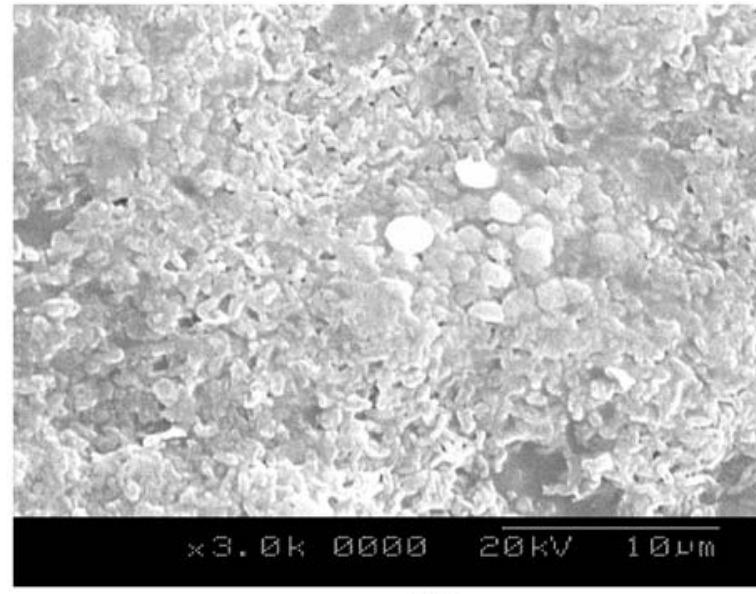

PU3

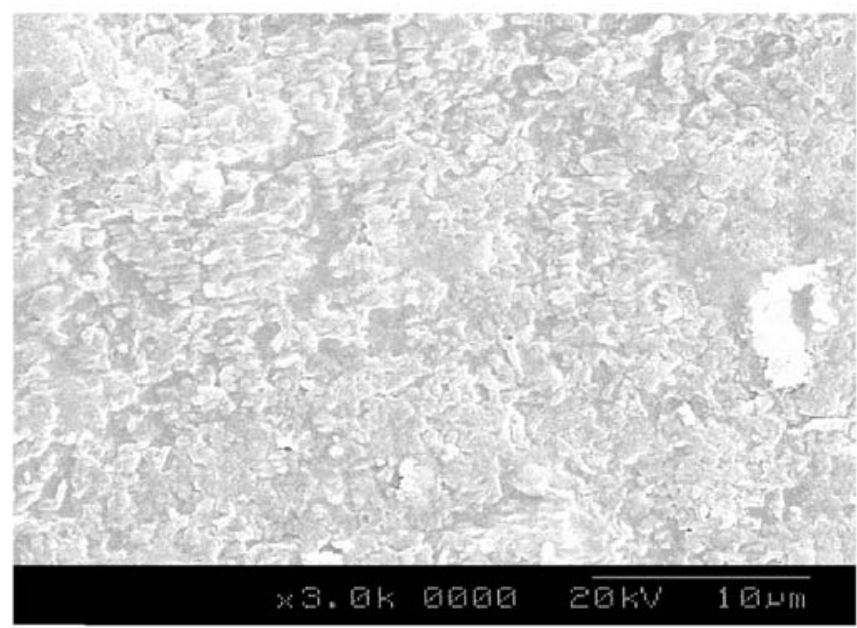

PU4

Figure 7. SEM images of degraded samples after 30 days of hydrolytic degradation 
urethane, i. e. PU1 followed by the slow degradation of hydrophobic and comparatively crystalline PCL based polyurethane, i. e. PU5.

The degradation pattern was examined for the blends and the pure polyurethanes by SEM and images of representative samples are shown in Figure 7. PU1 shows holes and crevices on a uniformly eroded surface as a result of both surface and bulk degradation while PU5 shows cracked and non-uniform surface structure due to predominantly surface erosion. PU1 is more hydrophilic due to the PEG soft segment while PU5 is hydrophobic due to the PCL soft segment. The SEM images of the blends PU2 and PU3 show that the blends are mainly degraded by surface erosion leading to spherulitic structures which are presumably formed by degradation of the crystalline PCL soft segment after degradation and dissolution of the PEG soft segment [15]. PU4, having a higher fraction of PCL based polyurethane, shows relatively uniform surface degradation.

\section{Conclusions}

Three blends of L-tyrosine based polyurethanes were fabricated by solvent evaporation from two polyurethanes having PEG and PCL as soft segments. Blending different compositions offers an easy technique to tune the properties of the parent L-tyrosine based polyurethanes. The wide variation in the properties of PEG and PCL based polyurethanes can be adjusted by changing the composition in order to obtain a suitable material for biomaterial applications. The spectral characterization of the blends indicates the relative compositions of these blends are close to theoretical ones. DSC study shows that blends with increasing PCL based polyurethane content are comparatively amorphous while contact angle measurements indicate that surfaces of these blends are mainly hydrophobic probably due to phase separated PCL based polyurethane. Blending of PCL based polyurethane with PEG based polyurethanes slightly enhanced the mechanical properties over the pure PEG material. The water absorption and degradation features along with the other characterization results of the blended polyurethanes show that these material properties can be easily controlled to fabricate a suitable material for different biomedical applications, particularly for fabrication of tissue engineering scaffold. These initial characterizations indicate that blends having lower content of PCL based polyurethane are more stable than blends having equal or higher PCL based polyurethane content. In general, the wide range of material properties of Ltyrosine based polyurethanes was achieved through a relatively simple fabrication technique.

\section{References}

[1] Stokes K., McVenes R., Anderson J. M.: Polyurethane elastomer biostability. Journal of Biomaterials Applications, 9, 321-354 (1995).

[2] Lamba N. M. K., Woodhouse K. A., Cooper S. L.: Polyurethanes in biomedical applications. CRC Press, New York (1998).

[3] Guan J., Sacks M. S., Beckman E. J., Wagner W. R.: Biodegradable poly(ether ester urethane)urea elastomers based on poly(ether ester) triblock copolymers and putrescine: synthesis, characterization and cytocompatibility. Biomaterials, 25, 85-96 (2004).

[4] Skarja G. A., Woodhouse K. A.: Synthesis and characterization of degradable polyurethane elastomers containing an amino acid-based chain extender. Journal of Biomaterials Science, Polymer Edition, 9, 271-295 (1998).

[5] Yeganeh H., Jamshidi H., Jamshidi S.: Synthesis and properties of novel biodegradable poly $(\varepsilon$-caprolactone)/poly(ethylene glycol)-based polyurethane elastomers. Polymer International, 56, 41-49 (2007).

[6] Buchanan C. M., Gedon S. C., White A. W., Wood M. D. E.: Cellulose acetate butyrate and poly(hydroxybutyrate-co-valerate) copolymer blends. Macromolecules, 25, 7373-7381 (1992).

[7] Shimamura E., Kasuya K., Kobayashi G., Shiotani T., Shima Y., Doi Y.: Physical properties and biodegradability of microbial of poly(3-hydroxybutyrate-co-3hydroxyhexanoate). Macromolecules, 27, 878-880 (1994).

[8] Sarkar D., Yang J-C., Sengupta A., Lopina S. T.: Synthesis and characterization of L-tyrosine based polyurethanes for biomaterial applications. Journal of Biomedical Materials Research, submitted (2007).

[9] Fromstein J. D., Woodhouse K. A.: Elastomeric biodegradable polyurethane blends for soft tissue applications. Journal of Biomaterials Science, Polymer Edition, 13, 391-406 (2002).

[10] Park T. W., Cohen S., Langer R.: Poly (L-lactic) acid/pluronic blends: characterization of phase separation behavior, degradation, and morphology and use as protein-releasing matrix. Macromolecules, 25, 116-122 (1992).

[11] Gan Z., Zhang J., Jiang B.: Poly( $\varepsilon$-caprolactone)/ poly(ethylene oxide) diblock copolymer II. Nonisothermal crystallization and melting behavior. Journal of Applied Polymer Science, 63, 1793-1804 (1997). 
[12] Olabisi O., Robenson L., Shaw M. T.: Polymer-polymer miscibility. Academic Press, New York (1979).

[13] Speckhard T. A., Cooper S. L.: Ultimate tensile properties of segmented polyurethane elastomers: factors leading to reduced properties for polyurethanes based on nonpolar soft segments. Rubber Chemistry and Technology, 59, 405-431 (1986).
[14] Averous L., Moro L., Dole P., Fringant C.: Properties of thermoplastic blends: starch-polycaprolactone. Polymer, 41, 4157-4167 (2000).

[15] Skarja G. A., Woodhouse K. A.: In vitro degradation and erosion of degradable segmented polyurethanes containing an amino acid-based chain extender. Journal of Biomaterial Science, Polymer Edition, 12, 851873 (2001). 